\title{
Peridynamics: Past, Present and Future
}

\author{
Erkan Oterkus ${ }^{1, a)}$ \\ ${ }^{1}$ PeriDynamics Research Centre, Department of Naval Architecture, Ocean and Marine Engineering, University of \\ Strathclyde, Glasgow, United Kingdom. \\ ${ }^{a)}$ Corresponding author: erkan.oterkus@strath.ac.uk
}

\begin{abstract}
Peridynamics is a new continuum mechanics formulation. It is especially suitable for predicting crack initiation and propagation in materials and structures. Its equations are in integro-differential equation form and do not contain spatial derivatives. Moreover, it allows non-local interactions between material points inside an interaction domain called horizon. In this study, a brief review of peridynamics is given describing its past progress, current status and potential future directions.
\end{abstract}

\section{INTRODUCTION}

Crack prediction is still a challenging area of solid mechanics although there are various methods available in the literature. Continuum mechanics is a widely used approach in solid mechanics to determine the response of a material or structure under external loading conditions. Continuum mechanics is based on the assumption that a structure is continuous as opposed to discrete atomic structure and it is composed of infinitely small volumes named as material points. Different continuum mechanics formulations exist and the most popular one was introduced by Cauchy almost two hundred years ago. According to Cauchy, a material point can interact with only the material points located in its nearest neighborhood. To represent the information transfer between material points, he defined traction vectors on the common surfaces between the material points which are in contact with each other. Moreover, he introduced an important parameter for each material point as stress. By using global balance of linear and angular momentum, and representing a relationship between the traction vector and stress, he obtained the equation of a material point located at $\mathbf{x}$ as

$$
\rho \ddot{\mathbf{u}}(\mathbf{x}, t)=\nabla \cdot \boldsymbol{\sigma}+\mathbf{b}(\mathbf{x}, t)
$$

where $\rho$ is the density, $\ddot{\mathbf{u}}$ is the acceleration, $\boldsymbol{\sigma}$ is the stress tensor, and $\mathbf{b}$ is the body load. Although Eq. (1) has been successfully used for the solution of numerous problems, it encounters a difficulty when cracks exist in the structure. Displacement field is discontinuous along the crack surfaces and because of this reason the spatial derivatives in Eq. (1) are not defined along the crack surfaces. To overcome this limitation, Silling [1] introduced a new continuum mechanics formulation called peridynamics. According to peridynamics, a material can interact with all material points inside the structure as opposed to Cauchy's formulation. The interaction between material points is represented by using peridynamic force. Therefore, the equation of motion of a material point in peridynamic theory can be expressed as 


$$
\rho \ddot{\mathbf{u}}(\mathbf{x}, t)=\int_{H}\left(\mathbf{f}-\mathbf{f}^{\prime}\right) d V^{\prime}+\mathbf{b}(\mathbf{x}, t)
$$

where $\mathbf{f}$ and $\mathbf{f}^{\prime}$ are the peridynamic forces between two interacting material points $\mathbf{x}$ and $\mathbf{x}^{\prime}$. Although a material point can interact with all material points in the structure, it is assumed that the interactions are limited to a region called horizon, $H$. Since the peridynamic equation given in Eq. (2) is integro-differential equation form and does not contain spatial derivatives, it is always valid regardless of discontinuities in the displacement field.

\section{PAST, PRESENT AND FUTURE OF PERIDYNAMICS}

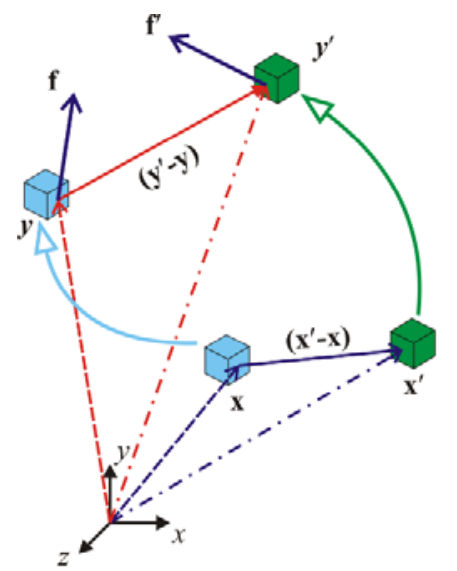

Non-ordinary state based PD Theory

(a)

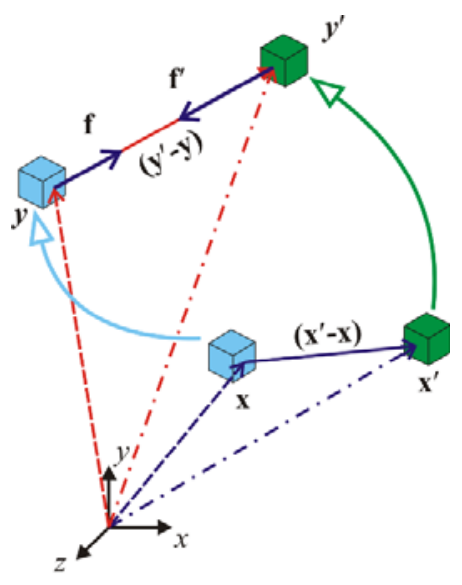

Ordinary state based PD Theory

(b)

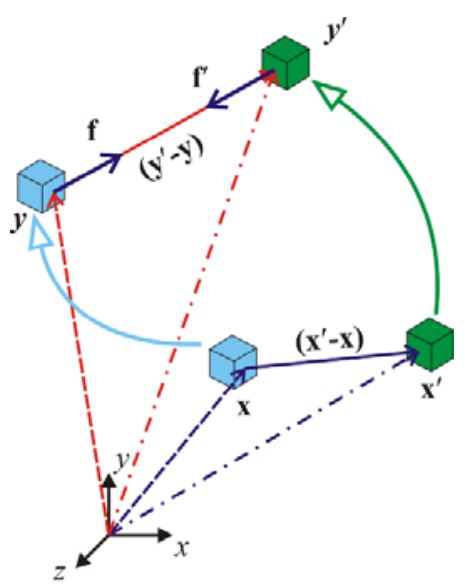

Bond based PD Theory

(c)

FIGURE 1. Different types of Peridynamic (PD) Theory; (a) Non-ordinary State Based PD Theory, (b) Ordinary State Based PD Theory, (c) Bond Based PD Theory

Depending on the peridynamic forces between material points, peridynamics is classified into different categories. As shown in Fig. (1a), if the peridynamic forces between two material points are in arbitrary direction, it is called as Non-ordinary State Based PD Theory. If it is assumed that the peridynamic forces are along the interaction direction but have different magnitudes, then it is called Ordinary State Based PD Theory (see Fig. 1b). If a further assumption is made by also enforcing the magnitudes of the forces are equal and they are in opposite direction, then it is called Bond Based PD Theory (see Fig. 1c). Bond Based PD Theory is the original PD formulation and it is simple to implement. However, it has limitation(s) on material constant(s). As explained in Madenci and Oterkus [2], PD equation of motion given in Eq. (2) can be derived by using Euler-Lagrange equations. PD theory has its own material parameters and these parameters can generally be expressed in terms of material constants of Cauchy's continuum mechanics formulation.

Definition of failure of peridynamics is pretty straightforward. For brittle materials, a stretch based failure criterion is widely used. According to this criterion, if the stretch between two material points exceeds a critical stretch value, the interaction is terminated. The critical stretch can be obtained from the critical energy release rate of the material.

The solution of PD equation of motion is generally obtained by using numerical techniques especially by using meshless method. The first PD research code, EMU, was developed at Sandia National Laboratories. It was then also implemented in a molecular dynamics code, LAMMPS, since peridynamics equations in discrete form are 
similar to molecular dynamics equations. It is also possible to implement peridynamics in commercial finite element software packages as explained in Macek and Silling [3].

Although peridynamics [4-9] was originally developed for solving mechanical field, it has been extended to other physical fields including thermal, moisture, porous flow, etc. [10-15]. It is also possible to use peridynamics for fluid flow analysis [16]. Peridynamics is not limited to isotropic materials and it can be used for the analysis of anisotropic materials [17-20] and materials at small scale such as graphene [21]. It is also suitable for fatigue [22] and impact analyses [23]. Peridynamic formulations for simplified structures such as beams, plates and shells are also available in the literature [24-30].

Peridynamics is a promising technique and it has a very good potential for many different applications. Potential future directions may include multiscale analysis, additive manufacturing, artificial intelligence and machine learning.

\section{CONCLUSIONS}

Peridynamics is a new continuum mechanics formulation. It is especially suitable for failure analysis of materials and structures due to its equations of motions which are integro-differential form and do not contain spatial derivatives. Peridynamic equations are generally solved numerically, especially by using meshless technique. It is also possible to implement peridynamics in commercial finite element software packages. Moreover, peridynamics is not limited to mechanical field, but can be utilized for the analysis of other physical fields. Therefore, peridynamics can be used as a multiphysics analysis tool with failure prediction capability. Potential future directions of peridynamics may include multiscale analysis, additive manufacturing, artificial intelligence and machine learning.

\section{ACKNOWLEDGEMENTS}

The author would like to thank Prof. Mas Irfan P. Hidayat, the chair of ICOMMET 2020 conference, for his kind invitation to the conference.

\section{REFERENCES}

1. S. A. Silling, J. Mech. Phys. Solids 48(1), 175-209 (2000).

2. E. Madenci and E. Oterkus, Peridynamic Theory and Its Applications (Springer, New York, 2014).

3. R. W. Macek and S. A. Silling, Finite Elem. Anal. Des. 43(15), 1169-1178 (2007).

4. M. Imachi, S. Tanaka, T. Q. Bui, S. Oterkus and E. Oterkus, Eng. Fract. Mech. 206, 359-374 (2019).

5. N. Zhu, D. De Meo and E. Oterkus, Mater. 9(12), 977 (2016).

6. M. F. Basoglu, Z. Zerin, A. Kefal and E. Oterkus, Comput. Mater. Sci. 162, 33-46 (2019).

7. B. Vazic, C. Diyaroglu, E. Oterkus, and S. Oterkus, Journal of Peridynamics and Nonlocal Modeling, 1-26 (2020).

8. E. Celik, E. Oterkus and I. Guven, Journal of Peridynamics and Nonlocal Modeling 1(1), 36-44 (2019).

9. O. Karpenko, S. Oterkus and E. Oterkus, Journal of Peridynamics and Nonlocal Modeling (2020).

10. H. Wang, E. Oterkus and S. Oterkus, Eng. Fract. Mech. 192, 176-191 (2018).

11. D. De Meo and E. Oterkus, Ocean Eng. 135, 76-83 (2017).

12. C. Diyaroglu, S. Oterkus, E. Oterkus and E. Madenci, IEEE Trans. Compon. Packag. Manuf. Technol. 7(11), 1823-1831 (2017).

13. H. Wang, E. Oterkus and S. Oterkus, Energies 11(6), 1461 (2018).

14. H. Wang, E. Oterkus, S. Celik and S. Toros, AIMS Energy 5(4), 585-600 (2017).

15. C. Diyaroglu, S. Oterkus, E. Oterkus, E. Madenci, S. Han and Y. Hwang, Microelectron. Reliab. 70, $103-111$ (2017). 
16. Y. Gao and S. Oterkus, Ocean Eng. 179, 135-158 (2019).

17. E. Oterkus and E. Madenci, "Peridynamics for failure prediction in composites," in 53rd AIAA/ASME/ASCE/AHS/ASC Structures, Structural Dynamics and Materials Conference 20th AIAA/ASME/AHS Adaptive Structures Conference 14th AIAA (2012), pp. 1692.

18. E. Oterkus, A. Barut and E. Madenci, "Damage growth prediction from loaded composite fastener holes by using peridynamic theory," in 51st AIAA/ASME/ASCE/AHS/ASC Structures, Structural Dynamics, and Materials Conference 18th AIAA/ASME/AHS Adaptive Structures Conference 12th (2010), pp. 3026.

19. Y. Gao and S. Oterkus, Compos. Struct. 207, 397-424 (2019).

20. S. Alpay and E. Madenci, Crack growth prediction in fully-coupled thermal and deformation fields using peridynamic theory," in 54th AIAA/ASME/ASCE/AHS/ASC structures, structural dynamics, and materials conference (2013), pp. 1477.

21. X. Liu, X. He, J. Wang, L. Sun, E. Oterkus, Proc. R. Soc. A 474(2217), 20180019 (2018).

22. Oterkus, E., Guven, I. and Madenci, E., "Fatigue failure model with peridynamic theory," in 12 th IEEE Intersociety Conference on Thermal and Thermomechanical Phenomena in Electronic Systems, (2010), pp. 16.

23. E. Oterkus, I. Guven, and E. Madenci, Open Eng. 2(4), 523-531 (2012).

24. Z. Yang, E. Oterkus, C.T. Nguyen and S. Oterkus, Contin. Mech. Thermodyn. 31(1), 301-315 (2019).

25. C. Diyaroglu, E. Oterkus and S. Oterkus, Math. Mech. Solids 24(2), 361-376 (2019).

26. Z. Yang, B. Vazic, C. Diyaroglu, E. Oterkus and S. Oterkus, Math. Mech. Solids 25(3), 727-738 (2020).

27. Z. Yang, E. Oterkus and S. Oterkus, Journal of Peridynamics and Nonlocal Modeling, 1-19 (2020).

28. Z. Yang, E. Oterkus, and S. Oterkus, Journal of Peridynamics and Nonlocal Modeling, 1-17 (2020).

29. C. T. Nguyen and S. Oterkus, Eng. Fract. Mech. 219, 106623 (2019).

30. C. T. Nguyen and S. Oterkus, Ocean Eng. 173, 244-267 (2019). 